Radiologe 2021 · 61:333-334

https://doi.org/10.1007/s00117-021-00832-4

Angenommen: 16. Februar 2021

(c) Springer Medizin Verlag GmbH, ein Teil von Springer Nature 2021

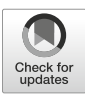

\title{
Marc-André Weber
}

Institut für Diagnostische und Interventionelle Radiologie, Kinder- und Neuroradiologie, Universitätsmedizin Rostock, Rostock, Deutschland

\section{Bildgebende Diagnostik der Hand - Teil 1}

Idee der Evolution Kreise zu ziehen, aber Bell dachte, eine nähere Betrachtung der Hand würde das dumme Geschwätz wohl beenden. „Die menschliche Hand“, schrieb er, „ist der letzte und beste Beweis für die göttliche Schöpfung“" [2].

Die Bildgebung der Hand fand de facto mit dem allerersten Röntgenbild durch Wilhelm Conrad Röntgen von der Hand seiner Frau Bertha am 22.12.1895 ihren Ursprung. Dem Radiologen kommt seit der Entstehung des Fachgebietes Radiologie eine Schlüsselposition zu bei der Erfassung und korrekten Beschreibung von Pathologien der Hand und der zeitnahen Befundkommunikation an den klinischen Partner. Auch in meiner Alma Mater Rostochiensis fand die Röntgentechnik bereits kurz nach der Entdeckung die ersten Anwendungen. Nach anfänglicher experimenteller Anwendung in der Physik wurde die Bildgebung bereits für die handchirurgische Diagnostik eingesetzt. Nach Durchführung einer Röntgenaufnahme (Expositionszeit von mehr als $15 \mathrm{~min}$ ) der linken Hand am 25.02.1896 konnte eine Revolverkugel in Projektion auf das Os metacarpale IV von dem hiesigen Chirurgie-Professor Carl Garré operativ entfernt werden [3].

Zurück zur Gegenwart: Das erste von zwei Themenheften zum Handgelenk und zur Hand startet mit einem Beitrag von Dr. Falko von Stillfried, Speyer, zum Thema „Was erwartet der Handchirurg vom Radiologen bei der bildgebenden Diagnostik der Hand?" Dr. von Stillfried betont insbesondere die Wichtigkeit einer zielgerichteten und klaren Kommunikation, angesichts der feinen und komplexen Strukturen an der Hand, zwischen behandelndem Arzt und Radiologen. Es folgt ein Beitrag von Prof. Dr.
Rainer Schmitt, München, und Kollegen zum Thema „Der ulnokarpale Komplex. Neue klinische und radiologische Überlegungen“. Die Autoren führen u.a. aus, dass bei ulnokarpalen Beschwerden ohne fokussierte Fragestellung des Zuweisers die Magnetresonanztomographie (MRT) als Diagnostik der Wahl empfohlen wird, bei explizierter Frage des Zuweisers nach dem Zustand der fovealen TFCC(triangulärer fibrokartilaginärer Komplex)-Lamina aber die direkte MR- oder CT-Arthrographie. Frau PD Dr. Jennifer Kollmer, Heidelberg, berichtet anschließend zum Thema „Worauf muss hinsichtlich der Nerven bei der Bildgebung der Hand geachtet werden?". Sie führt aus, dass mithilfe der bildgebenden Nervendarstellung durch Akquisition von spezialisierten, stark T2-gewichteten, fettsupprimierten Sequenzen in einem 3-Tesla-MR-System die hochauflösende Abbildung der peripheren Nerven bis hin zu den distalen Nervenendästen und Interdigitalnerven verlässlich möglich ist.

Das Themenheft wird von zwei Beiträgen von Prof. Dr. Matthias Bollow, Bochum, zu den klinischen Aspekten und zur Bildgebung der rheumatoiden Arthritis abgeschlossen. Alle rheumatischen Autoimmunerkrankungen gehen mit Arthritiden der Hände einher, wobei zwischen typischen und atypischen Arthritismustern der Hand differenziert werden kann, welche als Schlüssel zur Diagnose dienen. Die rheumatoide Arthritis als Prototyp der synovitischen Arthritiden weist typische Befallsmuster an der Hand auf. Mit Hilfe der MRT lassen sich bereits Frühstadien einer rheumatoiden Arthritis vor dem Auftreten von irreversiblen strukturelzes Buch: Die menschliche Hand und ihre Eigenschaften. $\mathrm{Zu}$ jener Zeit begann die 
len erosiven Schädigungen detektieren. Auch Erosionen lassen sich sensitiver als mit der Projektionsradiographie mittels MRT nachweisen.

Insgesamt hoffe ich, dass Ihnen, liebe Leserinnen und Leser, dieses erste der beiden Themenhefte bereits einen umfassenden Überblick über die aktuellen diagnostischen Herausforderungen bei diesem speziellen Thema der Radiologie der Hand liefert, der durch das zweite Themenheft in der nächsten Ausgabe von Der Radiologe komplettiert wird.

Ich wünsche Ihnen sehr viel Spaß beim Lesen und hoffe, Sie finden diese Zusammenstellung interessant und hilfreich für Ihre praktische Tätigkeit.

Mit herzlichen Grüßen,

Ihr

Marc-André Weber

\section{Korrespondenzadresse}

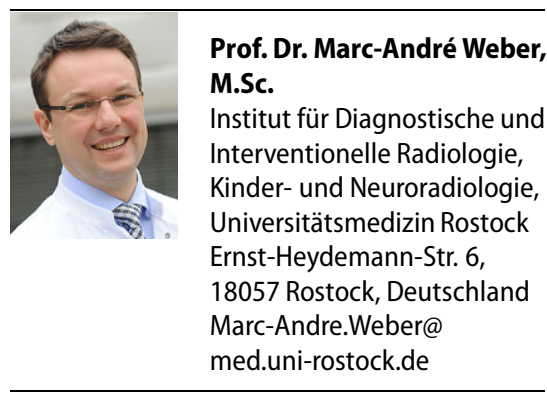

Interessenkonflikt. M.-A. Weber gibt an, dass kein Interessenkonflikt besteht.

\section{Literatur}

1. Zimmer C (2012) Wie die Evolution die menschliche Hand schuf. https://www.welt. de/wissenschaft/article106233333/Wie-dieEvolution-die-menschliche-Hand-schuf.html (Erstellt:27.Apr. 2012).Zugegriffen:30.Dez. 2020

2. Sir Bell C (1836) Die menschliche Hand und ihre Eigenschaften. Aus dem Englischen des Sir Charles Bell von Dr. Hermann Hauff. Neff, Stuttgart

3. Weber M-A, Kolbe L, Sänger $P$, Mundelsee J, Burmeister J (2019) Die Exponate der Rostocker Radiologiesammlung. Übersichts- und Informationsbooklet, S46 (www.med.uni-rostock.de)

\section{Das Geheimnis der Muskelzelle}

Eine neue Studie am Max-Delbrück-Centrum für Molekulare Medizin (MDC) untersucht die unterschiedlichen Kerne einer Muskelzelle und hilft so unter anderem dabei, Muskelerkrankungen wie die Duchenne-Dystrophie besser zu verstehen.

Inwieweit sich die Kerne einer einzelnen Muskelfaser hinsichtlich ihrer Genaktivität voneinander unterscheiden und welche Auswirkungen das auf die Funktion des Muskels hat, war bislang kaum bekannt. Hier setzt eine neue MDC-Studie an: Wie im Fachblatt "Nature Communications" berichtet, wurde die Genexpression solcher Zellkerne mit einem noch recht neuen Verfahren namens Einzelkern-RNASequenzierung untersucht. Dabei stieß das Forschungsteam um Professorin Carmen Birchmeier auf eine unerwartet hohe Vielfalt genetischer Aktivität.

Untersucht wurden zunächst mehrere tausend Kerne aus gewöhnlichen Muskelfasern von Mäusen sowie Kerne aus Muskelfasern, die sich nach einer Verletzung regenerierten. Um herauszufinden, ob sich die Genaktivität zwischen dem ruhenden und dem wachsenden Muskel unterscheidet, wurden die Kerne genetisch markiert und aus den Zellen isoliert. Das Team entdeckte dabei in beiden Sorten von Muskelfasern sehr viele unterschiedliche Typen von Kernen und auch ganz neue Arten spezialisierter Kerne mit jeweils eigenen Mustern der Genaktivität.

In einem nächsten Schritt wurden die Kerne aus Muskelfasern von Mäusen mit Duchenne-Dystrophie, der häufigsten Form des erblichen Muskelschwunds beim Menschen, untersucht. Hier war ein Verlust vieler Zellkerntypen zu beobachten, andere Typen waren nicht mehr in Clustern organisiert, sondern über die ganze Zelle verstreut. Darüber konnten krankheitsspezifische Subtypen von Zellkernen gefunden werden: Zum einen Kerne, die Gene nur noch in geringem Umfang ablasen und gerade abstarben, zum anderen solche, in denen jene Gene besonders aktiv waren, die beschädigte Muskelfasern reparieren. Solche vermehrten Genaktivitäten konnten auch in Muskelbiopsien von Patienten mit Muskelerkrankungen beobachtet werden - offenbar versucht der Muskel, auf diese
Weise den krankheitsbedingten Schäden entgegenzusteuern.

Mithilfe der MDC-Studie können also pathologische Mechanismen im Muskel untersucht und der Erfolg neuer Therapieansätze überprüft werden. Da Funktionsstörungen des Muskels auch bei vielen anderen Krankheiten wie beispielsweise Diabetes sowie beim alters- und krebsbedingten Muskelschwund zu beobachten sind, lässt sich der Ansatz zudem dazu nutzen, auch derartige Veränderungen besser zu erforschen.

Das Max-Delbrück-Centrum für Molekulare Medizin in der HelmholtzGemeinschaft (MDC) wurde 1992 in Berlin gegründet. Aufgabe des MDC ist die Erforschung molekularer Mechanismen, um Krankheitsursachen auf den Grund zu gehen und damit eine bessere und wirksamere Krankheitsdiagnose, -prävention und behandlung zu ermöglichen.

\author{
Quelle: Max-Delbrück-Centrum für \\ Molekulare Medizin in der Helmholtz- \\ Gemeinschaft Berlin, \\ www.mdc-berlin.de
}

